\title{
Biomechanics-based graph matching for augmented CT-CBCT
}

\author{
Jaime Garcia Guevara ${ }^{1,2} \quad$ Igor Peterlik $^{1}$ \\ Marie-Odile Berger ${ }^{1,2} \quad$ Stephane Cotin ${ }^{1}$ \\ Received: date / Accepted: date
}

\begin{abstract}
Purpose: Augmenting intraoperative cone beam computed tomography (CBCT) images with preoperative computed tomography (CT) data in the context of imageguided liver therapy is proposed. The expected benefit is an improved visualization of tumor(s), vascular system and other internal structures of interest.

Method: An automatic elastic registration based on matching of vascular trees extracted from both the preoperative and intraoperative images is presented. Although methods dedicated to non-rigid graph matching exist, they are not efficient when large intraoperative deformations of tissues occur, as is the case during the liver surgery. The contribution is an extension of the graph matching algorithm using Gaussian process regression (GPR) [1]: First, an improved GPR matching is introduced by imposing additional constraints during the matching when the number of hypothesis is large; like the original algorithm, this extended version does not require a manual initialization of matching. Second, a fast biomechanical model is employed to make the method capable of handling large deformations.

Results: The proposed automatic intraoperative augmentation is evaluated on both synthetic and real data. It is demonstrated that the algorithm is capable of handling large deformations, thus being more robust and reliable than previous approaches. Moreover, the time required to perform the elastic registration is compatible with the intraoperative navigation scenario.
\end{abstract}

Conclusion: A biomechanics-based graph matching method, which can handle large deformations and augment intraoperative CBCT, is presented and evaluated.

\section{Introduction}

Cone beam computed tomography (CBCT) is an accessible imaging technique in which the X-ray tube and detector panel rotate around the patient. Although it is easier to deploy than CT or MRI in an operating room. CBCT has about half contrast to noise 
ratio compared to $\mathrm{CT}$, besides $\mathrm{CBCT}$ is affected by noise artifacts, scatter, partial volume effects, beam hardening, ring effects and motion artifacts [2]. Therefore, certain lesions and important structures are not visible in CBCT images. This problem can be addressed by augmenting intraoperative images with preoperative data in order to compensate for their lack of detail. This can lead to a significantly improved visualization for the clinician.

The principal clinical application of this work is Transarterial chemoembolization (TACE), a palliative treatment for hepatocellular carcinoma, one of the most common cancers in the world. TACE is a percutaneous treatment performed under radiological guidance. It uses the difference in blood supply between the liver tumor and healthy liver parenchyma to (partially) destroy the tumor by implanting a cytotoxic agent inside the hepatic artery followed by embolization of the hepatic artery [3]. During the procedure, fluoroscopic images or CBCT images are acquired while a catheter is navigated towards the tumor through the abdominal aorta and hepatic arteries. As a result, contrast-enhanced CBCT images, showing a partial vascular tree, are acquired. Registering this partial graph with the complete tree obtained from the preoperative CT has the potential to enhance the visualization, speed up navigation to the target with reduced number of $\mathrm{CBCT}$ acquisitions.

Image registration is an active area of research in medical imaging. When deformable organs are considered, registration requires to define a deformation model, as well as an objective criteria which quantifies the alignment between the target and the source images [4]. In the case of the liver, the objective criteria is mostly based on the registration of the hepatic vessel network visible in both pre and intraoperative modalities. For highly deformable organs, the choice of deformation is essential for the registration procedure. Simple models as thin-plate splines transformations [5] allow to reach computational efficiency through mean square estimation when minimizing the objective criterion, but do not ensure that the deformation is physically coherent with the organ properties. Conversely, models with large degrees of freedom may lead to inappropriate or unnatural motion of the organs. In addition, optimization is more challenging and may be highly dependent on initial conditions. Realistic organ deformation using a mass-spring vessels model have been done [6], unfortunately it required a manual initialization. Preoperative and simulated intraoperative (random noise and downsampling) CT image were registered using a biomechanical insufflation model and an intensity based optimization [7]. However the insufflation induced deformation might not work in the general case and the intensity based optimization is affected by real CBCT poor image quality.

A recent contribution, proposed by Seradell et al. [1], is to perform nonrigid graph registration using Gaussian process regression (GPR) whose predictions are progressively refined as more correspondences are added. These predictions are used to explore the set of correspondences starting with the most likely ones. This method has shown to be effective on various examples, does not require an initialization step and is able to cope with partial matches, topological differences and deformation. However, the nonparametric mapping induced by the Gaussian process may lead to incomplete solutions since GPR is not able to cope with large non-linear deformations. In addition, the computational cost is prohibitive for large graphs. This led the authors to propose a method based on Monte Carlo tree search [8] which balances exploring new possible 
matches and extending existing matches. This method is suited to graphs consisting of vertices connected with curves and the deformation model is only defined implicitly, controlling the relative distance changes with a parameter. Large and nonuniform deformations can thus hardly be handled by this method correctly.

This contribution builds on the work of Seradell et al. [1] and proposes a graph matching method able to cope with large deformations, as those which appear in hepatic surgery, due to respiratory motion or organ manipulation. The central idea of this paper is to combine a GPR matching with a biomechanical model of the organ. GPR allows for rigorous and fast error propagation but is extremely versatile, while biomechanical transformations are slower to compute but provide physically correct hypotheses. Integrating the two approaches allows us to significantly improve the quality of the registration for moderate or large organ deformations while reducing computation times.

\section{Method}

In this section, the main steps of the method are presented in detail. Although the method is assessed on liver, most of the steps presented below are directly applicable to any vascularized organ. Initially, the vascular trees are segmented from both the preoperative computed tomography angiography (CTA) and the intraoperative CBCT images using model-based vessel detection [9]. The tree topologies composed of nodes (bifurcations) and edges are extracted from the segmented binary images using an algorithm based on on Dijkstra minimum-cost spanning tree [10]. The branches are smoothed with a Bézier curve allowing for sampling points with arbitrary density along segments between two bifurcations.

The building blocks of this method for robust graph matching are: (i) An improved version of the GPR matching (iGPR) based on the original GPR matching [1]. iGPR algorithm reduces the set of hypotheses explored, thus decreasing significantly the time needed to perform the matching. (ii) A biomechanical graph matching (BGM) capable of retrieving all the matches including those which suppose large tissue deformations. The BGM matching employs the results of iGPR as an initialization. (iii) A final alignment step based on the biomechanical model (fineBGM).

The original GPR graph matching method [1] is briefly summarized in section 2.1.

The iGPR algorithm is presented in section 2.2 followed by the biomechanical model description (section 2.3), and its deployment in the registration method BGM (section 2.4). The overview of the method is depicted in Fig. 1.

\subsection{Gaussian process graph matching}

The graph matching algorithm [1] relies on Gaussian process regression (GPR) which is a nonparametric kernel-based probabilistic model used to compute a smooth geometric mapping. GPR priors embody beliefs about typical properties of the shape and the kernel $k\left(x, x^{\prime}\right)$ specifies the correlation of two function values corresponding to two different inputs $x$ and $x^{\prime}$ [11]. It does not require a manual initialization and handles partial matching and topological differences. 


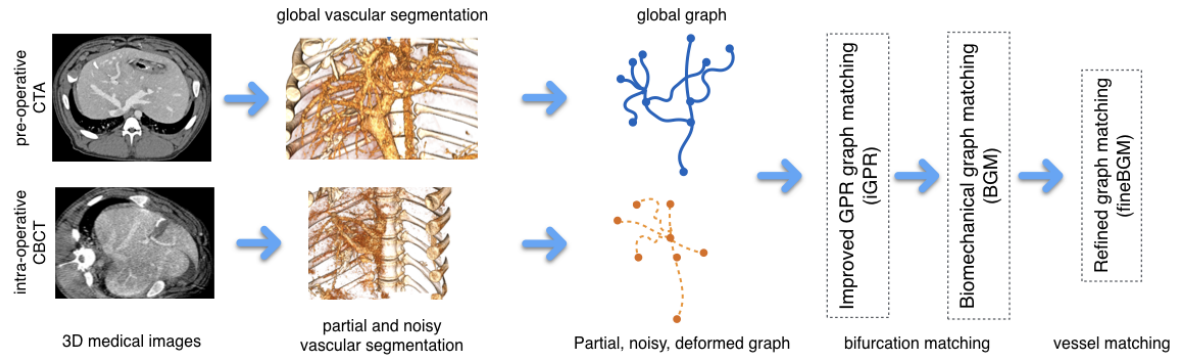

Figure 1: The pipeline is automatic: starting from preoperative CTA and CBCT image, the vessels are segmented and graphs of the vascular system extracted. An improved GPR graph matching is used to compute, between the 2 graphs, an initial bifurcations match. This first match is used to initialize the BGM, providing a coarse matching. Then a fine FEM alignment (fineBGM) of all the graph points is performed .

To facilitate the presentation of this contribution, the original graph matching algorithm is presented in Alg. 1 where it is applied to find correspondences between two graphs $\mathscr{G}^{A}=\left(\vec{X}^{A}, \vec{E}^{A}\right)$ and $\mathscr{G}^{B}=\left(\vec{X}^{B}, \vec{E}^{B}\right)$ where for $I \in\{A, B\}$ :

$\vec{X}^{I}=\left\{\mathbf{x}_{1}^{I}, \ldots, \mathbf{x}_{n_{I}}^{I}\right\}$ is the set of graph nodes (tree bifurcations),

$\vec{E}^{I}=\left\{e_{1}^{I}, \ldots, e_{m_{I}}^{I}\right\}$ is the set of graph edges.

The GPR hyperparameters [11] and precision of the measurement noise are denoted as $\Theta$ and $\beta$, respectively.

The algorithm recursively constructs a set of hypotheses, where each hypothesis $\pi_{t}$ is a set of source and target bifurcation matching pairs $\mathbf{x}_{i}^{A} \leftrightarrow \mathbf{x}_{j}^{B}$, starting with a random hypothesis (line 1). Given the nature of GPR, these correspondences are used to predict that a point $\mathbf{x}_{i}^{A}$ in a correspondence with $\mathbf{x}_{j}^{B}$ can be found at a location with mean $m$ and a covariance $\sigma^{2}$ estimated by the GPR; this operation is performed by procedure ComputeMapping (line 3) of Alg. 1; the reader is referred to [1] for further details. A quality of each generated hypothesis is determined by the number of inliers (line 4) as

$$
S_{\pi}=|I| \quad \text { where } \quad I=\left\{e^{B}\left|\exists m_{\pi}\left(e^{A}\right), H \cdot\right| m_{\pi}\left(e^{A}\right)-e^{B} \mid<\beta^{-1 / 2}\right\}
$$

and $H$ is the Hungarian algorithm assignment.

Further, for each unmatched bifurcation from graph $\mathscr{G}^{A}$, a bounded region $\mathscr{B}_{i}$ is computed (line 6) as

$$
\mathscr{B}_{i}=\left\{\forall \mathbf{x}_{j}^{B} \in \vec{X}^{B} \mid M^{2}\left(m_{\pi_{t}}\left(\mathbf{x}_{i}^{A}\right), \mathbf{x}_{j}^{B}\right)<t_{M A H}\right\}
$$

where $M^{2}=\left(m_{\pi_{t}}\left(\mathbf{x}_{i}^{A}\right)-\mathbf{x}_{j}^{B}\right)^{T}\left(\sigma_{\pi_{t}}^{2}\left(\mathbf{x}_{i}^{A}\right)\right)^{-1}\left(m_{\pi_{t}}\left(\mathbf{x}_{i}^{A}\right)-\mathbf{x}_{j}^{B}\right)$ is the Mahalanobis distance. The bounded region $\mathscr{B}_{i}$ that has the lowest number of potential candidates indicates the next bifurcations pairs which are added to the actual hypothesis. With the new hypothesis $\pi_{t}$ as parameter, the method is called recursively until no more matches are found. When this happens, the algorithm backtracks to the previous hypothesis, explores an alternative candidate match and continues the recursion until the limit of candidates to explore is reached. Finally, when the recursion is completed, the hypothesis with 
the maximum number of inliers is selected. In the original algorithm this hypothesis is used to initialize an iterative fine-matching step which matches the graph points in between bifurcations using the Hungarian algorithm assignment as long as the inlierbased metric improves.

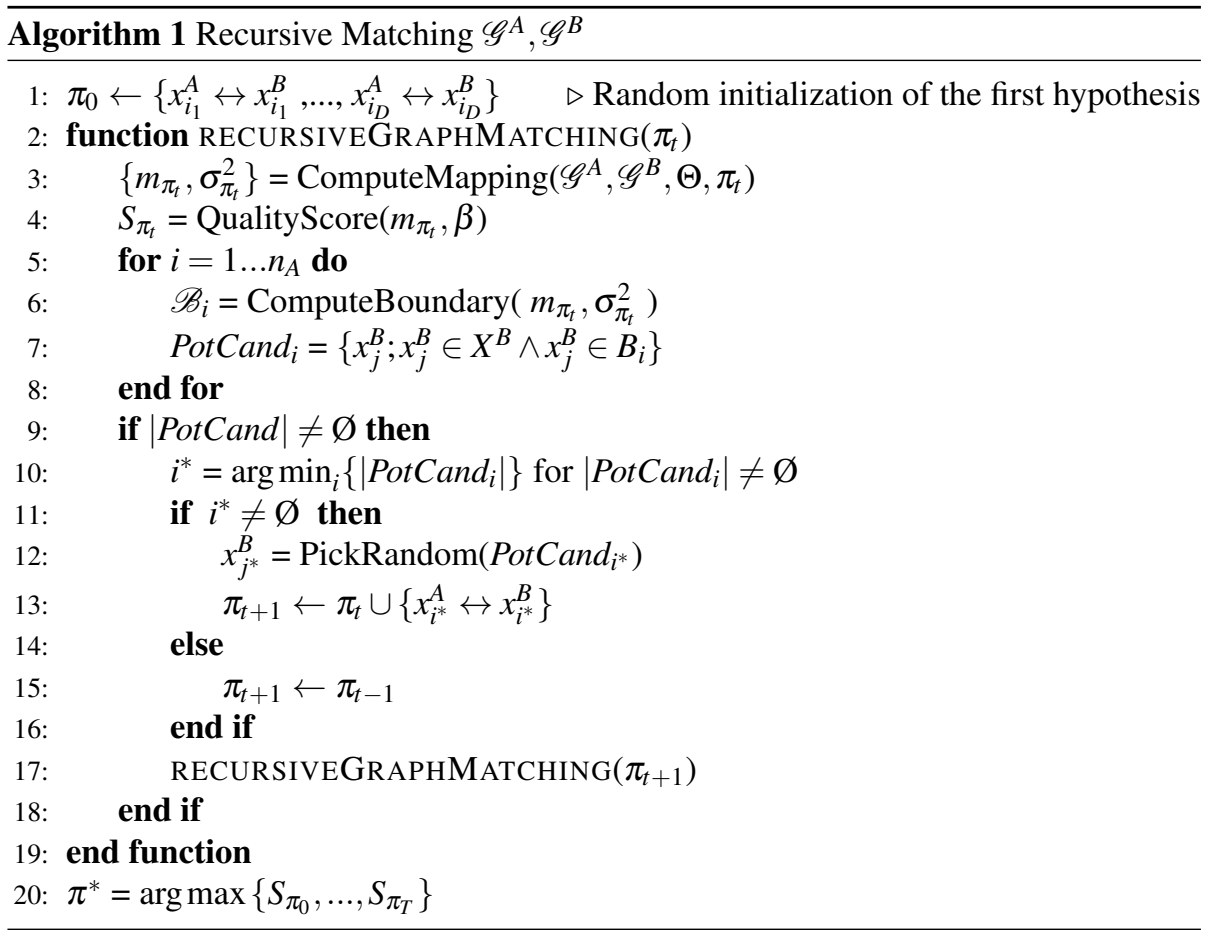

The original matching algorithm has two principal limitations: practically, when more than 25 bifurcations are considered, the time needed to perform the matching does not comply with intraoperative timing constraints. Second, the GPR smooth geometrical mapping is not capable of finding correct matches when large nonlinear deformations occur. These limitations are addressed in following sections.

\subsection{Improved Gaussian process graph matching}

In this section three improvements over the original GPR method are described aiming to reduce the computation time. Their common objective is to avoid the exploration of hypotheses when the matched bifurcations radii and tree topology are highly different. And when the GPR covariance is highly unreliable.

Radius constraint. At every bifurcation the radius of each vessel is averaged over the segment of the vessel between the bifurcation and $20 \%$ of the length of the vessel. To assign the branch correspondences, the target $\left(r_{v}\right)$ and source $\left(r_{u}\right)$ averaged radii of each bifurcation are sorted. Theoretically, the radii should be similar, however, to take into account possible inaccuracies in the segmentation, bifurcations are considered for 
matching if they are below a threshold $t_{R}$ :

$$
\left|\frac{\min \left(r_{u}, r_{v}\right)}{\max \left(r_{u}, r_{v}\right)}-1.0\right|<t_{R} .
$$

Topological constraints. In the case where the target and model graphs have similar structures, topological properties of tree branching patterns of the trees can be used to discard erroneous matching hypotheses. Two topological characteristics are used: the Horton-Strahler number $(H N)$ of a node and the centrifugal order of a segment. The centrifugal order $(\mathrm{CO})$ of a segment denotes its topological distance to the root segment [12], whereas the $H N$ of a node or a tree is a numerical measure of its branching complexity [13]. The $H N$ of each graph is here normalized with respect to its maximum. Two thresholds $t_{C O}$ and $t_{H N}$ are specified to discard from the matching hypotheses trees which have too different topological properties. Bifurcations are considered for matching if:

$$
\left|C O_{u}-C O_{v}\right|<t_{C O} \text { and }\left|H N_{u}-H N_{v}\right|<t_{H N}
$$

These constraints are used when the number of bifurcations is bigger than 30 in order to reduce the space of possible combinations. Initialization is done using only root bifurcations which are the ones with higher $H N$ and big vessels mean radius. Once initialized, the complete set of bifurcations is used in the next steps. These constraints allow to dramatically decrease the matching time, however, the trees must have a similar structure. They are especially used in real data (section 3.2). The typical values used for the constraints thresholds are: $t_{R}=0.4, t_{C O}=4$ and $t_{H N}=0.6$.

Half first covariance constraint. Looking at the behavior of GPR based matching, the covariance evaluated with a small number of matched bifurcations largely exceeds the motion that the organ can undergo during deformation, thus leading to exploration of many false hypotheses at the beginning of the matching process. To handle this issue the threshold $t_{M A H}$ used to define the bounded region (Eq. 2), is halved when the hypothesis to explore has less than 4 bifurcations matched.

\subsection{Fast biomechanical liver model}

Depending on the values of the hyperparameters, the original GPR matching [1] is flexible and through the kernel, it may theoretically adapt to large range of deformations. Nevertheless, the deformations which occur inside the soft tissues during surgical manipulations display high level of nonlinearity due to complex properties of tissues. For example, the presence of pathology or other internal structures may introduce heterogeneity and anisotropy of the deformation field. Therefore, the GPR matching is extended with a fast biomechanical model of soft tissue which increases the robustness and performance of matching since it is capable of handling large nonlinear deformations while preserving their physical nature.

The biomechanical finite element (FE) model of liver [14] used makes the assumption of linear stress-strain relation, it employs a co-rotational formulation of the strain to handle large nonlinear displacements. The model reconstructed from patient-specific 
anatomy is reliable for physics-based augmented reality [15]. The proposed matching method is not limited to the chosen FE model which can be replaced by another elastic model, including non-linear ones [16].

The FE model is used in a displacement-zero traction scenario. In this case, no forces are applied to the model, however, the deformation is induced by non-homogeneous Dirichlet boundary conditions. In case of homogeneous isotropic model, the resulting deformation does not depend on the mechanical parameters which are usually not know accurately due to the patient-specific modeling [17].

The FE mesh of liver composed of linear tetrahedra has been generated [18] from a 3D mask segmented from the preoperative image. Moreover, it allows to specify a set of points located inside the mask which become nodes of the mesh. A matching hypothesis $\pi_{t}$ is given by pairs of bifurcations $\mathbf{x}_{i}^{A} \leftrightarrow \mathbf{x}_{j}^{B}$. As the positions in set $\vec{X}^{A}$ defined in section 2.1 are known preoperatively, the mesh is generated so that each point from this set coincides with a node $n_{i}$ of the FE mesh. In this case, the deformation of the model is driven by the set of non-homogeneous Dirichlet conditions where for each matching pair, the displacement of node $n_{i}$ is prescribed as $\mathbf{u}_{n_{i}}=\mathbf{p}_{i}=\mathbf{x}_{j}^{B}-\mathbf{x}_{i}^{A}$.

The Dirichlet conditions are imposed via penalty method: physically, this method can be interpreted as adding a set of elastic linear springs which pull each node $n_{i}$ from its initial position $\mathbf{x}_{i}^{A}$ to the target position $\mathbf{x}_{j}^{B}$. Similarly, the deformation is computed as a dynamic process given by the system $\mathbf{M} \ddot{\mathbf{u}}+\mathbf{B} \dot{\mathbf{u}}+\mathbf{K}(\mathbf{u})=\mathbf{p}$ where $\mathbf{M}$ is a mass matrix, $\mathbf{K}$ is the nonlinear co-rotational stiffness with contributions from elastic springs, $\mathbf{B}$ is a damping matrix approximated using Rayleigh stiffness $r_{K}$ and Rayleigh mass $r_{M}$ as $\mathbf{B}=r_{M} \mathbf{M}+r_{K} \mathbf{K}$ and $\mathbf{p}$ is a vector gathering the prescribed displacements given by the actual matching pairs [15]. The system is integrated by implicit Euler method with single linearization per integration step with $r_{K}=r_{M}=0.1$. The simulation is completed as soon as the simulation achieves the static equilibrium.

\subsection{Biomechanics-based graph matching and fine alignment}

The matching using the FE transformation is described in Alg. 2. It replaces the mean from the original GPR matching with the biomechanical model transformation (line 4). While the physically plausible FE transformation avoids the exploration of false hypotheses and finds more correct hypothesis, it is too computationally expensive to be employed from the beginning of the matching. On average, the time required to compute the FE transformation is 1000 times larger than the time required by the GPR. Therefore, instead of the random initialization, the FE-based matching is initialized with the result on iGPR (line 1), thus providing, within a feasible time, a correct but often incomplete set of matched bifurcations considering small or medium-sized deformations. Starting from this solution, the BGM is able to find the matches that were not discovered with the iGPR, while meeting the time constraints.

The FE model-based fine-alignment (fineBGM) step is similar to fineGPR [1] but replaces the mean mapping by a nonrigid transformation using the liver biomechanical model. It also removes the matched graph points that are closer than the maximal tetrahedral size of the FEM mesh. 


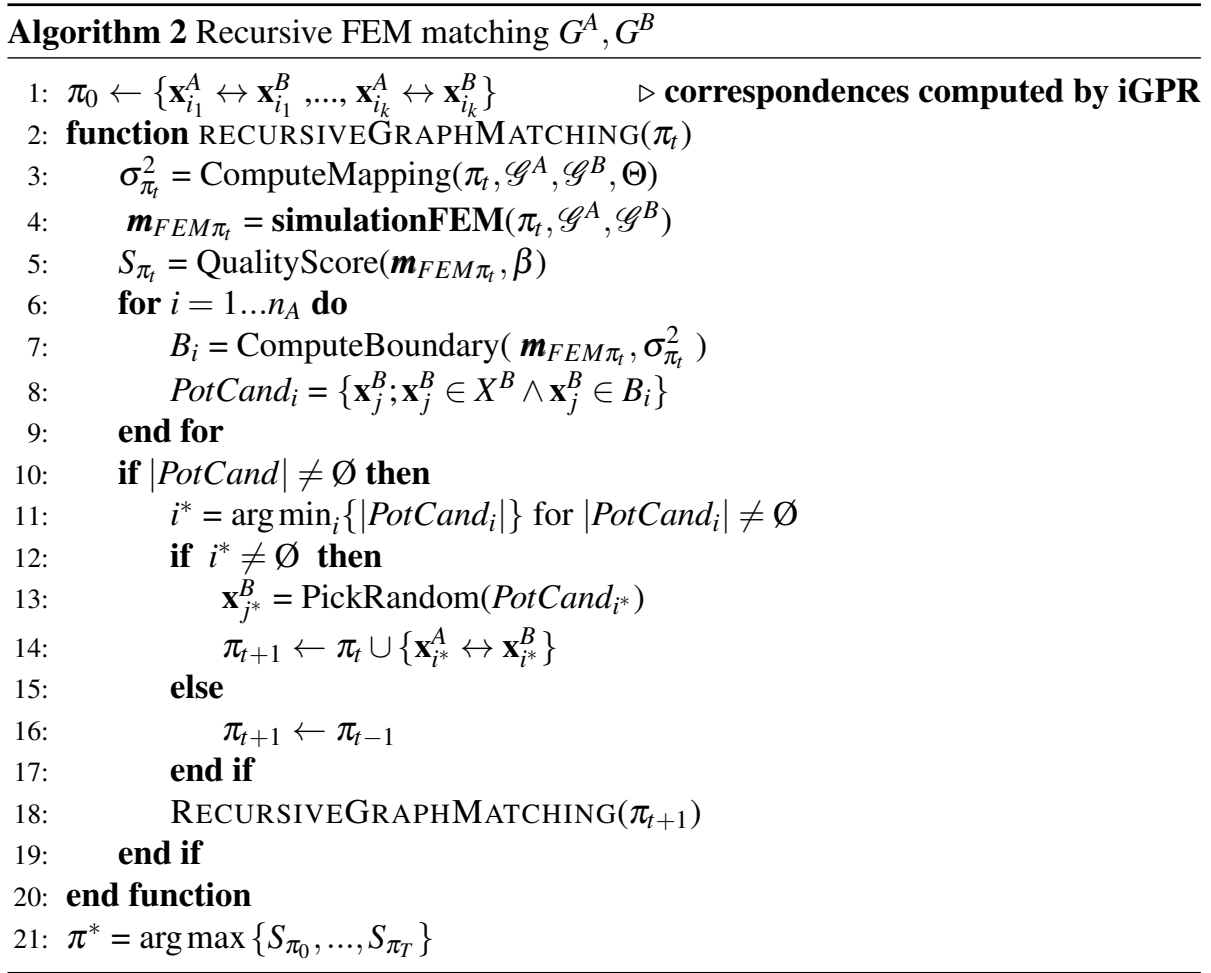

\section{Experiments and results}

The developed methods were evaluated on both synthetic and real data.

\subsection{Experiments on synthetic data}

First simulated realistic deformations and vessels removal was done on the CT graphs to resemble CBCT. This ground truth data is used to evaluate and compare the matching methods described.

Ground truth simulated data. The liver FEM and portal vein tree used for validation are extracted from the IRCAD human dataset ${ }^{1}$. A StVenant Kirchhoff consitutive model is used to simulate realistic deformations of the liver model. Constant pressure is applied to the model to simulate a pneumoperitoneum. Dirichlet boundary conditions are set near the portal vein. Fig. 2 illustrates the simulation setup. Three different levels of deformations were simulated, using the same pressure value $(1.6 \mathrm{kPa})$ and varying Young modulii $(15.0 \mathrm{kPa}, 7.0 \mathrm{kPa}$ and $3.5 \mathrm{kPa})$ leading to small, medium and large displacement fields. The medium deformation is shown in Fig. 2.b. The original graph is in green and the deformed graph is color mapped with the deformation displacement. It is important to note that the FEM model used for matching is linear

${ }^{1}$ https://www.ircad.fr/research/3dircadb/ 
which makes the computation faster and uses a $1.5 \mathrm{kPa}$ Young Modulus which is different from the ground truth simulations. In order to simulate segmentation problems due to poor image quality, $40 \%$ random leaf branches were removed.

(a)

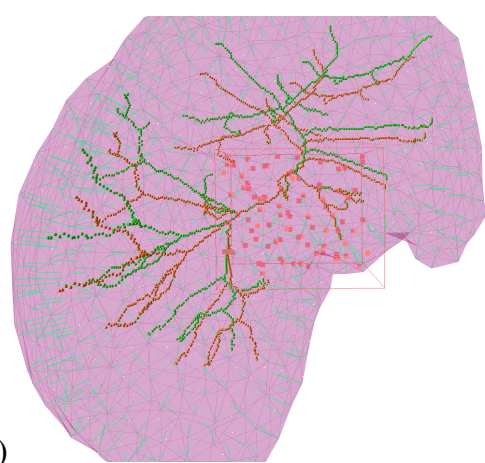

(b)

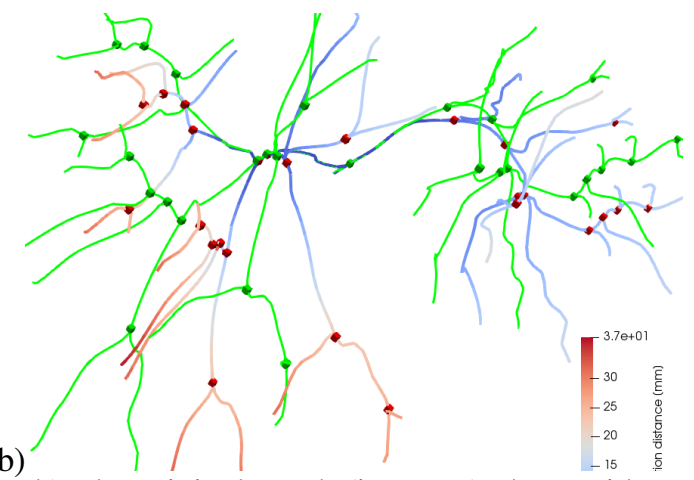

Figure 2: a) Medium deformation setup. b) The original graph (in green) along with the deformation distance color mapped graph.

Evaluating the original and iGPR algorithms. In Fig. 3, the original GPR algorithm (in cyan) is compared against the iGPR (in blue) and the Half first covariance improvement shown (in purple). The GPR algorithm depends on many internal parameters such as $t_{M A H}$ (equation (2)), the geodesic threshold $G$ (defines the candidate matches' maximum geodesic distance difference) and the outlier tolerance $O$ (determines the number of bifurcations within $\mathscr{B}_{i}$ to explore before stopping). So the algorithms are evaluated with representative commonly used [1] parameters. The target registration error (TRE) is plotted in solid lines whereas the timing is indicated with dashed lines. The improved versions are up to 1000 times faster than the original one with similar TRE. The TRE is computed on every edge point in the original graph, including the $40 \%$ removed vessel branches. The green thick horizontal line marks the ground truth coarse matching TRE, it is computed using the ground truth bifurcation matches in the target and model graphs. Given that $40 \%$ target bifurcations were removed, coarse ground truth TRE using only bifurcations does not reaches zero.

Comparing the iGPR and the BGM method The efficiency of the BGM method for medium and large deformations is proved in this experiment. The TRE is used to measure the coarse matching accuracy. A second metric used is the convex hull volume of the matched bifurcations. The convex hull volume gives a better idea of the global matching; a bigger volume ensures that the computed deformation is valid on a larger domain. Fig. 4.a shows the TRE of the iGPR and the BGM on the commonly used parameters for the medium deformation transformation. As can be seen on the figure, the results of the iGPR step is dependent on the parameters whereas the results of the BGM are more accurate $(4.2 \mathrm{~mm}$ against $12.2 \mathrm{~mm}$ ) and limitedly dependent on the parameters used in the first iGPR step. Times required by the two processes are shown in the graph (time scale is on the right of the figure). In addition, much more bifurcations are matched with BGM and the convex hull volume of the set of matched bifurcations (Fig. 4.b) is larger for the BGM whereas the iGPR method tends to provide only incomplete solution sets which are in fact only a partial graphs match. This is an important strength of the BGM algorithm. Note that large deformation matchings give 


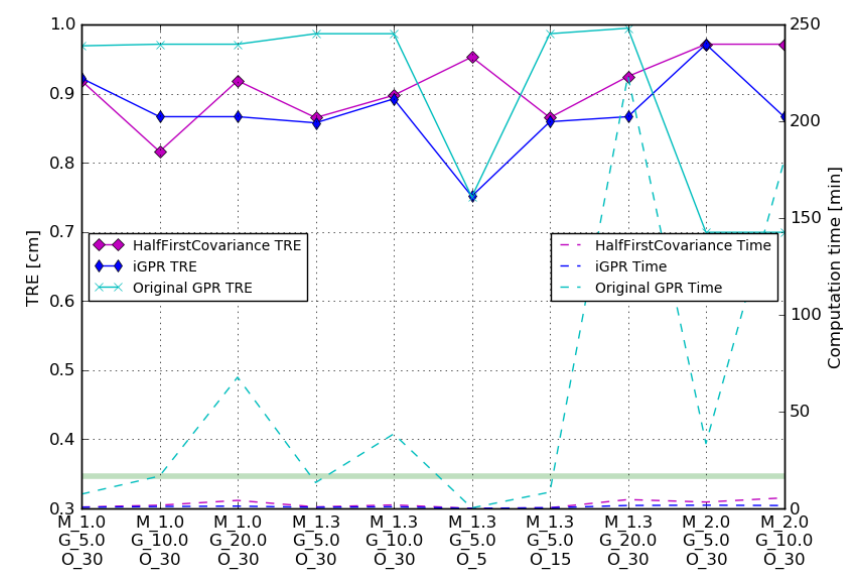

Figure 3: Comparison of original GPR (cyan), Half first covariance (purple) and iGPR (blue) matching methods. The TRE are in solid lines whereas the times are in dashed lines. The proposed GPR improvements (Half first covariance and iGPR) have similar TRE while being much faster original GPR matching [1].

similar results, summarized in Fig. 6.

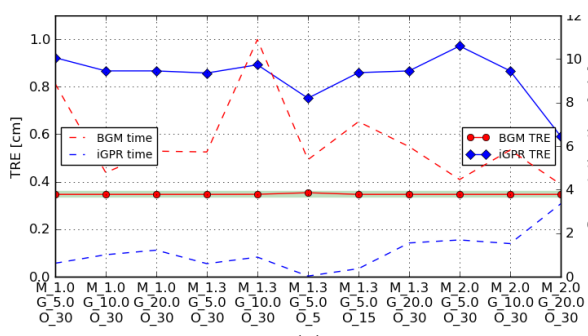

(a)

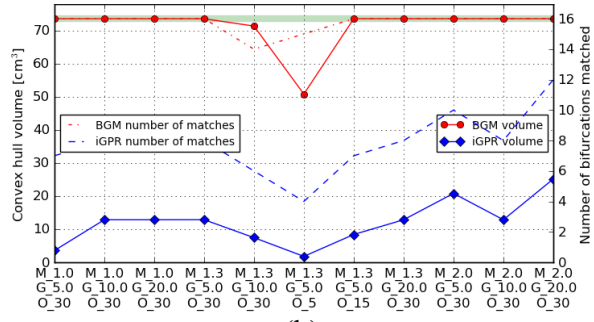

(b)

Figure 4: Comparison of iGPR with BGM. a) The TRE is plotted in solid lines. The iGPR (in blue) although is fast it is far from the ground truth (green thick horizontal line) and has high variability on the parameters used. The BGM TRE (in red) converges to the coarse TRE ground truth. b) Similarly, the BGM convex hull volume and its number of bifurcations matches mostly converge to the ground truth values.

The Fig. 5 compares TRE and timing of the fineGPR and the fineBGM matching methods on the medium deformation. Here again, the TRE is noticeably smaller with the fineBGM matching and the results are largely independent of the parameters. Note that fineGPR has small computation times because it uses the iGPR matching.

The Fig. 6 summarizes the results on the three synthetic deformations. It shows a significant TRE improvement and less sensitiveness to parameters of the fineBGM compared to the fineGPR method. These result are specially important in the medium and large deformations. Still the fineBGM keeps computation times within an acceptable intraoperative tolerance. 


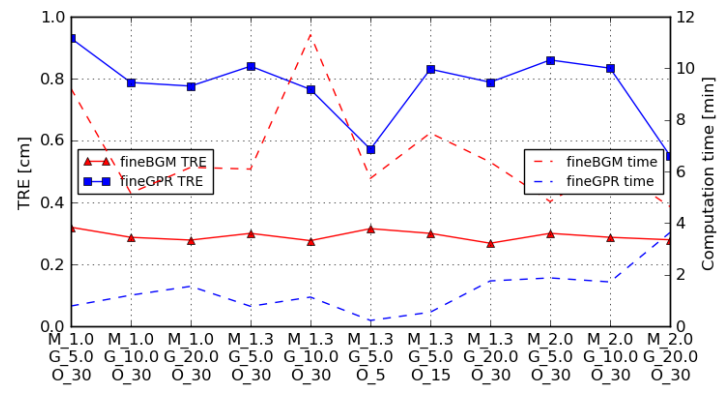

Figure 5: Comparison of fineGPR with fineBGM graph matching methods. The TRE is plotted in solid lines. The fineGPR (in blue) is faster but has bigger TRE $(8 \mathrm{~mm})$ and has high variance depending on the parameters used. The fineBGM TRE (in red) only has $3 \mathrm{~mm}$ error with small dependence on the parameters.

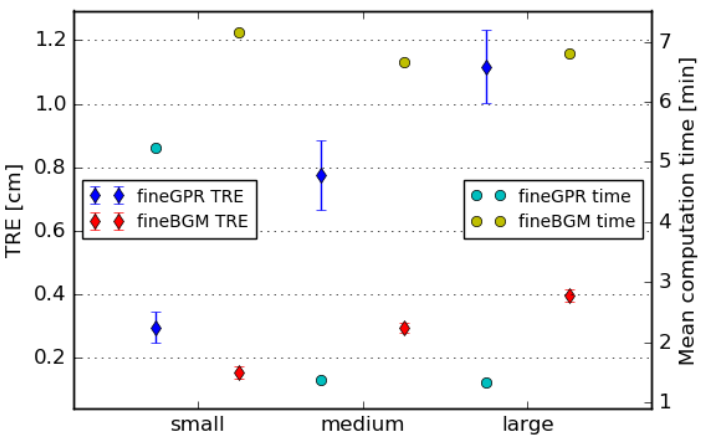

Figure 6: The fineGPR is faster but has bigger TRE and high variance specially in the medium and large deformations. The TRE and dependence on the parameters in the fineBGM is small and it has an acceptable computation time increase. 


\subsection{Experiments on real data}

Experiments were done on two real porcine data. CT was acquired preoperatively in supine position whereas CBCT was acquired after insufflation on flank position, both images were acquired with contrast agent injection. The portal vein is visible in both modalities so it is used for registration. However less vessel branches are visible in CBCT compared CT (see Fig. 1). And due to image noise several false branches are segmented in CBCT. The hepatic vein is only visible in CT and is an important vessel augmented in CBCT (transparent blue vein in Figs. 7.b and 8.c ).

For the first porcine data, the Fig. 7 shows the registration of the two vessel trees after a rigid registration (a) and the fineBGM registration (b). In the CT image 50 bifurcations (green cubes) are extracted while 46 (pink cubes) in CBCT. Although both modalities have similar number of bifurcations, several false CBCT bifurcations are due to noise (non matched pink cubes in Fig. 7.b). The topological constraints described in Section 2.2 are used to reduce the computation time. In total there are 19 correct matched bifurcations (purple cubes). Three artificial tumors were inserted in the porcine liver to measure the registration accuracy. The tumors centroids are used to compute TRE and they are shown with red and green spheres. The two trees are globally well registered except a vessel (Fig. 7.b) which is not transformed well because some bifurcations are missing in the target segmentation. Despite the differences between the trees, the superposition of the tumors is very good. The BGM method has a TRE of $5.6 \mathrm{~mm}$ and the fineBGM TRE goes down to $5.0 \mathrm{~mm}$. While only using fineGPR matching has a TRE of $13.7 \mathrm{~mm}$. The computation time on a regular desktop computer (Intel Core i7-4790K CPU @ 4.00GHz, GeForce GTX 970) was 3.8 seconds for the iGPR plus 21.4 minutes for the BGM and fineBGM. The matching is implemented in single thread and could be parallelized if the explored hypotheses are tracked in shared memory.

In order to evaluate the influence of the segmentation on the matching process, the Table 1 shows the matching error obtained when varying the segmentation parameters. In this experiment only the left partial side of the tree shown in Fig. 7, found in the three segmentations, was used. As can be seen, though the number target bifurcations varies from 16 to 32 , the TRE varies from 5.0 to $6.4 \mathrm{~mm}$ and is thus only slightly dependent on the segmentation. Still the matching time is dependent on the number of bifurcations and consequently on the segmentation.

Table 1: TRE for three segmentations.

\begin{tabular}{lllll}
\hline Segmentation & Bifurcations' number & Matches' number & TRE [mm] & Matching time [min] \\
\hline 1 & 32 & 14 & $5.0 \pm 0.6$ & 7.3 \\
2 & 16 & 9 & $5.8 \pm 1.3$ & 2.7 \\
3 & 27 & 13 & $6.4 \pm 0.6$ & 6.8 \\
\hline
\end{tabular}

A second porcine experiment is shown in Fig. 8. The acquisition and deformation conditions were similar to the previous experiment. But this CBCT image is much noisier and only few vessels are visible and segmented. The target CBCT graph has 36 bifurcations but several false bifurcations (non matched pink cubes in Fig. 8.b) are due to noise. Only a partial model CT graph with 34 bifurcations was used for matching. Six inserted landmarks were used to evaluate the matching, resulting in $4.0 \pm 2.1 \mathrm{~mm}$ 

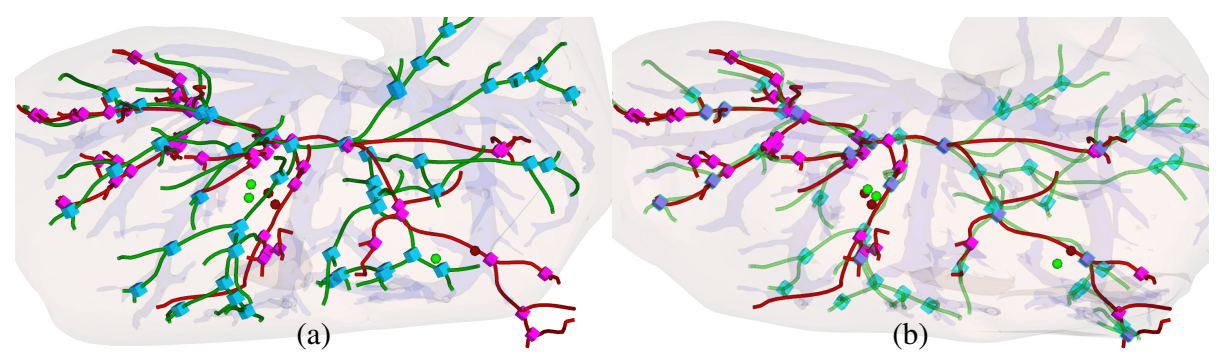

Figure 7: The target CBCT and model CT graphs plotted, respectively, in red and green. Similarly the bifurcations are shown with cubes and three inserted landmarks centroids with spheres. The augmented hepatic vein shown in transparent blue. The model is rigidly aligned (a) to get an idea of the deformation. The fineBGM matching (b) shows matched bifurcations with purple cubes.

TRE. The time needed for the iGPR is 2.4 minutes plus 3.7 minutes for the BGM and fineBGM. Finally, the CBCT view augmented with the CT vessel tree is showed in Fig. 8.c.
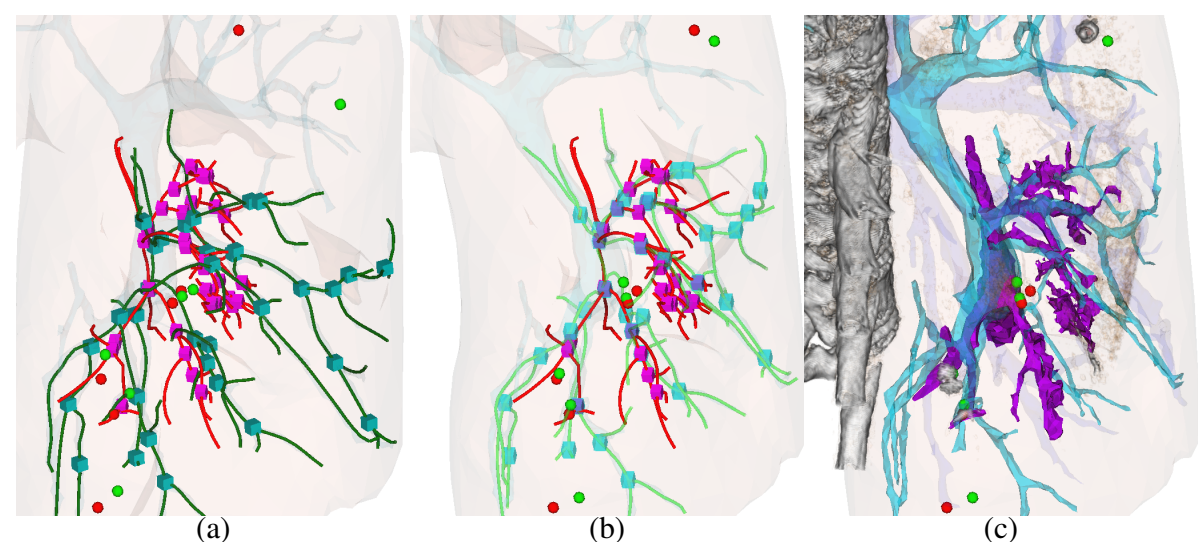

Figure 8: The target and model graphs plotted, respectively, in red and green. Similarly the bifurcations are shown with cubes and six inserted landmarks centroids with spheres. The model is rigidly aligned (a). The fineBGM matching (b) shows matched bifurcations with purple cubes. An augmented view with the target and model portal vein in purple and blue respectively (c).

\section{Conclusion}

The proposed biomechanics-based graph matching outperforms existing methods both in terms of accuracy and by its ability to handle large deformations. With respect to the original method, the time remains within acceptable intraoperative constraints and could be improved with dedicated implementation.

Since the matching time is directly linked to the number of vascular trees bifurcations, the surgeon could choose automatic segmentation parameters that result in fewer 
bifurcations but is still appropriate to the clinical gesture. This will impact the matching time.

The method relies on the definition of several parameters. Some of them related to the biomechanichal model, while others have geometrical nature. At this stage the method is more sensitive to the geometrical parameters, than the biomechanical ones. So some tunning when using real data still remains necessary for optimal results.

Besides improving the surgeon's visualization of the target and risk structures, augmenting non visible intraoperative structures like small vessels and tumors, could allow to reach them during TACE, or other image guided procedures. Demonstrated on CT/CBCT porcine vessels matching, this work paves the way towards augmented reality applications on highly deformable organs. In addition, no constraints on the deformation are imposed and the matching is only based on vessels, thus making the method able to work with imagery of different natures. According to the clinical gesture, other constraints (visible anatomical landmarks such as the liver surface) could be used in the registration.

Acknowledgments The authors are grateful for the support from Inria, the MIMESIS and MAGRIT teams, and IHU Strasbourg.

Funding Jaime Garcia Guevara is supported by the Grand Est region and Inria.

Compliance with ethical standards All institutional and national guidelines for the care and use of laboratory animals were followed.

Conflict of interest Jaime Garcia Guevara, Igor Peterlik, Marie-Odile Berger, and Stephane Cotin declare that they have no conflict of interest.

Ethical approval Animal data was collected under institutionally approved animal ethics protocol.

Informed consent This articles does not contain patient data.

\section{References}

[1] Serradell, E., Pinheiro, M.A., Sznitman, R., Kybic, J., Moreno-Noguer, F., Fua, P.: Non-rigid graph registration using active testing search. IEEE Trans Pattern Anal Mach Intell. 37(3), 625-638 (2015)

[2] Tacher, V., Radaelli, A., Lin, M., Geschwind, J.F.: How i do it: Cone-beam ct during transarterial chemoembolization for liver cancer. Radiology 274(2), 320334 (2015)

[3] European Association For The Study Of The Liver: EASL-EORTC clinical practice guidelines: management of hepatocellular carcinoma. Journal of hepatology 56(4), 908-943 (2012)

[4] Sotiras, A., Davatzikos, C., Paragios, N.: Deformable medical image registration: A survey. IEEE Trans Med Imag 32(7), 1153-1190 (2013)

[5] Lange, T., Papenberg, N., Heldmann, S., Modersitzki, J., Fischer, B., Lamecker, H., Schlag, P.M.: 3D ultrasound-CT registration of the liver using combined landmark-intensity information. Int J Comput Assist Radiol Surg. 4(1), 79-88 (2009) 
[6] Dagon, B., Baur, C., Bettschart, V.: Real-time update of 3D deformable models for computer aided liver surgery. 19th Int. Conf. on Pattern Recognition pp. 2-5 (2008)

[7] Oktay, O., Zhang, L., Mansi, T., Mountney, P., Mewes, P., Nicolau, S., Soler, L., Chefd'hotel, C.: Biomechanically driven registration of pre- to intra-operative $3 \mathrm{~d}$ images for laparoscopic surgery. In: Int. Conf. MICCAI, pp. 1-9. Springer (2013)

[8] Pinheiro, M.A., Kybic, J., Fua, P.: Geometric graph matching using monte carlo tree search. IEEE Trans Pattern Anal Mach Intell. 39(11), 2171-2185 (2017)

[9] Smistad, E., Elster, A.C., Lindseth, F.: GPU accelerated segmentation and centerline extraction of tubular structures from medical images. Int J Comput Assist Radiol Surg. 9(4), 561-575 (2014)

[10] Plantefève, R., Kadoury, S., Tang, A., Peterlik, I.: Robust Automatic GraphBased Skeletonization of Hepatic Vascular Trees, pp. 20-28. LNCS, volume 10552, Springer International Publishing (2017)

[11] Rasmussen, C., Williams, C.: Gaussian processes for machine learning. MIT Press (2006)

[12] van Pelt, J., Verwer, R.W.H., Uylings, H.B.M.: Centrifugal-order distributions in binary topological trees. Bulletin of Mathematical Biology 51(4), 511-536 (1989)

[13] Devroye, L., Kruszewski, P.: A note on the Horton-Strahler number for random trees. Information Processing Letters 2(56), 95-99 (1995)

[14] Peterlík, I., Duriez, C., Cotin, S.: Modeling and real-time simulation of a vascularized liver tissue. In: Int. Conf. MICCAI, pp. 50-57. Springer (2012)

[15] Plantefève, R., Peterlik, I., Haouchine, N., Cotin, S.: Patient-specific biomechanical modeling for guidance during minimally-invasive hepatic surgery. Ann Biomed Eng. 44(1), 139-153 (2016)

[16] Marchesseau, S., Chatelin, S., Delingette, H.: Non linear Biomechanical Model of the Liver. In: Y. Payan, J. Ohayon (eds.) Biomechanics of Living Organs, p. 602. Elsevier (2017)

[17] Wittek, A., Hawkins, T., Miller, K.: On the unimportance of constitutive models in computing brain deformation for image-guided surgery. Biomech model mechanobiol. 8(1), 77-84 (2009)

[18] Boltcheva, D., Yvinec, M., Boissonnat, J.D.: Mesh generation from 3D multimaterial images. In: Int. Conf. MICCAI, pp. 283-290. Springer (2009) 